\title{
The Ejection of Runaway Massive Binaries
}

\section{Virginia McSwain ${ }^{1}$, Scott M. Ransom ${ }^{2}$, Tabetha S. Boyajian ${ }^{3}$, Erika D. Grundstrom ${ }^{3}$, and Mallory S.E. Roberts ${ }^{4}$}

\author{
${ }^{1}$ NSF Astronomy and Astrophysics Postdoctoral Fellow; \\ Department of Astronomy, Yale University, P.O. Box 208101, \\ New Haven, CT 06520-8101, USA, email: mcswain@astro.yale.edu \\ ${ }^{2}$ National Radio Astronomy Observatory, 520 Edgemont Road, \\ Charlottesville, VA 22903, USA, email: sransom@nrao.edu \\ ${ }^{3}$ Department of Physics and Astronomy, Georgia State University, \\ P.O. Box 4106, Atlanta, GA 30302-4106, USA, \\ email: tabetha@chara.gsu.edu, erika@chara.gsu.edu, gies@chara.gsu.edu \\ ${ }^{4}$ Eureka Scientific, Inc., 2452 Delmer Street Suite 100, \\ Oakland, CA 94602-3017, USA, email: malloryr@gmail.com
}

\begin{abstract}
The runaway O-type stars HD 14633 and HD 15137 are both SB1 systems that were probably ejected from the open cluster NGC 654 . Were these stars dynamically ejected by close gravitational encounters in the dense cluster, or did the binaries each receive a kick from a supernova in one member? We present new results from our investigation of the optical, $\mathrm{X}$-ray, and radio properties of these binary systems to discuss the probable ejection scenarios. We argue that these binaries may have been ejected via dynamical interactions in the dense cluster environment.
\end{abstract}

Keywords. binaries: spectroscopic, stars: early-type, stars: kinematics, stars: winds, stars: individual (HD 14633, HD 15137), pulsars: general, X-rays: binaries

\section{Introduction}

Most O- and B-type stars form in open clusters and stellar associations, but a small fraction are observed at high galactic latitudes and with large peculiar space velocities. These runaway stars were likely ejected from the clusters of their birth, either by close multi-body interactions in the dense cluster environment or by supernovae explosions in close binaries. Identifying the dominant scenario producing runaway stars can offer important clues to the evolution of close binary stars and open clusters.

Distinguishing between the dynamical or supernova ejection mechanisms among runaway spectroscopic binaries can be a difficult task since both are expected to produce eccentric, relatively short period binaries (Leonard \& Duncan 1990; Portegies Zwart 2000). Only dynamical interactions are expected to produce double-lined spectroscopic binaries (SB2s), while a single-lined system (SB1) may be formed either way. It can be nearly impossible to detect a cool, low mass, optical companion with an O- or B-type primary, while a neutron star companion might be identified either as a pulsar or by $\mathrm{X}$-ray emission produced during mass accretion.

Spectroscopic investigations of HD 14633 and HD 15137 have found short orbital periods and low mass companions (Boyajian et al. 2005; McSwain et al. 2006). HD 14633 was ejected from the open cluster NGC 654 about 14 Myr ago, and HD 15137 was ejected from the same cluster about $10 \mathrm{Myr}$ ago (Boyajian et al. 2005). Presumably, both systems obtained their high runaway velocities during a supernova explosion in a close binary, and the $\mathrm{O}$ stars remain bound to the stellar remnant. In this work, we 

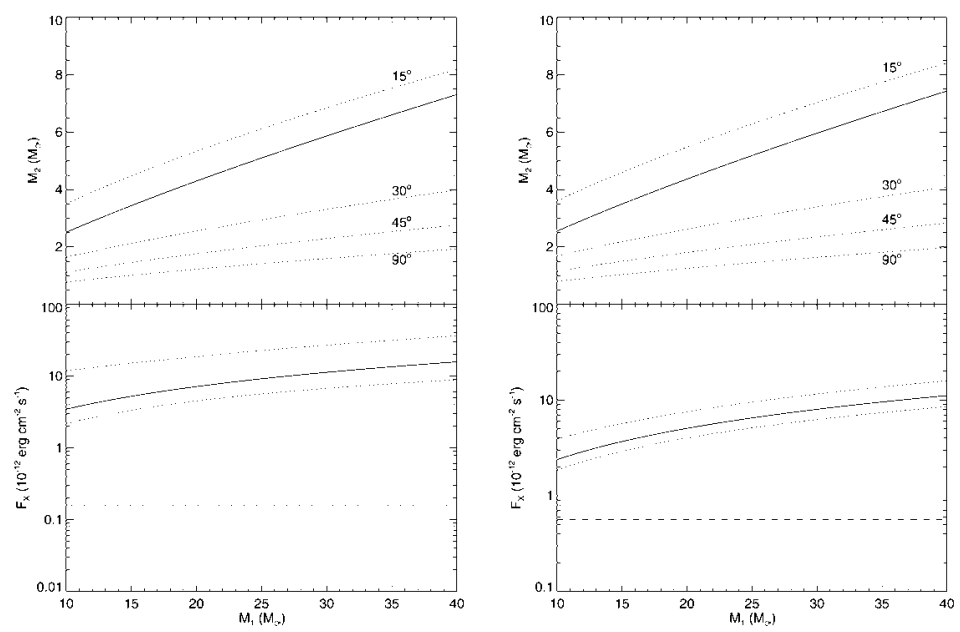

Figure 1. (top) Mass diagram for a range of inclination angles (dotted lines) and the most probable companion mass (solid line), based on the statistical method of Mazeh \& Goldberg (1992). (bottom) The predicted time-averaged X-ray flux (solid line) compared to the observed upper limit of $F_{\mathrm{X}}$ from $R O S A T / \mathrm{PSPC}$ observations (dashed line). This prediction assumes the minimum value of $M_{2}$, and a higher mass companion will produce an even greater $F_{\mathrm{X}}$. The left and right plots show the relationships for HD 14633 and HD 15137, respectively.

test this hypothesis using a collection of new observations and archival data across the electromagnetic spectrum.

\section{Pulsar Search Results}

Although previous searches have failed to detect radio pulsars in runaway OB stars (Philp et al. 1996; Sayer et al. 1996), we performed a new, more sensitive search to investigate the companions of both HD 14633 and HD 15137. Our search offers several advantages over these earlier searches: our new orbital ephemerides (McSwain et al. 2006) allowed us to schedule observations near the time of apastron, we used higher frequencies that are more likely to detect radio pulses dispersed by the stellar winds, and we obtained a better flux density sensitivity than previous searches.

We observed both targets using the National Radio Astronomy Observatory's 100m Green Bank Telescope and the Pulsar Spigot back-end, and the data were reduced using the PRESTO software package (Ransom 2001). No pulsars were detected in either HD 14633 or HD 15137.

\section{Predicted X-ray Luminosity from a Wind Accretion Model}

If HD 14633 and HD 15137 do contain compact companions, should these stars have detectable X-ray emission? We predict the X-ray flux of each quiet MXRB candidate using the wind accretion model of Lamers et al. (1976), modified for eccentric orbits. In Figure 1, the predicted fluxes are compared to the estimated upper limits for their unabsorbed X-ray fluxes using other detected sources from the ROSAT All-Sky Survey and pointed ROSAT observations within a 30' radius of each star (White et al. 2000; Voges et al. 2000). We predict X-ray fluxes of HD 14633 and HD 15137 that are an order of magnitude greater than the observed upper limits. However, the lack of X-ray detections does not conclusively rule out neutron stars. 
The conditions for wind accretion onto a neutron star depend strongly upon the spin rate and the magnetic field (Lipunov 1992). A young neutron star in the ejector or propellor regimes will not accrete significant amounts of material because its fast rotation and/or large magnetic field sweep material out of the system at a distance larger than the accretion capture radius, $R_{\mathrm{a}}$. As the neutron star spins down over time, the corotation radius, $R_{\mathrm{c}}$, becomes larger than both the magnetospheric radius, $R_{\text {mag }}$, and $R_{\mathrm{a}}$. We estimate these values for the critical distances in our systems: $R_{\mathrm{a}} \sim 10^{10} \mathrm{~cm}, R_{\mathrm{c}} \sim 10^{8}-$ $10^{9} \mathrm{~cm}$, and $R_{\text {mag }} \sim 10^{9} \mathrm{~cm}$. Unrestrained accretion requires $R_{\text {mag }}<R_{\mathrm{a}}$ and $R_{\mathrm{mag}}<R_{\mathrm{c}}$, which may be possible in these systems. However, even a non-accreting neutron star could be detected by its thermal or non-thermal X-ray spectrum (Lipunov 1992). Further X-ray observations are required to confirm or refute the presence of neutron stars in HD 14633 and HD 15137.

\section{Conclusions}

If HD 14633 and HD 15137 were ejected by supernovae in these close binary systems, they should contain neutron stars detectable as either radio pulsars and/or X-ray sources. Our search for pulsars with the Green Bank Telescope revealed no detections, and the predicted X-ray emission from wind accretion is much larger than the observed limits. Furthermore, neither system exhibits an infrared excess from an accretion disk around a compact companion (McSwain et al. 2006). While a neutron star cannot be ruled out, the probable companion masses (Figure 1) support higher mass objects. Therefore we argue that these binaries do not contain neutron stars, and we suggest that they may have been ejected from NGC 654 by dynamical interactions in the dense cluster environment.

\section{Acknowledgements}

M. V. M. gratefully acknowledges travel support from NRAO, and she is supported by an NSF Astronomy and Astrophysics Postdoctoral Fellowship under award AST0401460. This material is based on work supported by the National Science Foundation under Grants No. AST-0205297 and AST-0506573 (D. R. G.). Institutional support has been provided from the GSU College of Arts and Sciences and from the Research Program Enhancement fund of the Board of Regents of the University System of Georgia, administered through the GSU Office of the Vice President for Research.

\section{References}

Boyajian, T.S., Beaulieu, T.D., Gies, D.R., Huang, W., McSwain, M.V., Riddle, R.L., Wingert, D.W., \& De Becker, M. 2005, ApJ, 621, 978

Lamers, H.J.G.L.M., van den Heuvel, E.P.J., \& Petterson, J.A. 1976, A\&A, 49, 327

Leonard, P.J.T. \& Duncan, M.J. 1990, AJ, 99, 608

Lipunov, V.M. 1992, Astrophysics of Neutron Stars (Berlin: Springer-Verlag)

Mazeh, T. \& Goldberg, D. 1992, ApJ, 394, 592

McSwain, M.V., Boyajian, T.S., Grundstrom, E.D., \& Gies, D.R. 2006, ApJ, in press (astro$\mathrm{ph} / 0608270)$

Philp, C.J., Evans, C.R., Leonard, P.J.T., \& Frail, D.A. 1996, AJ, 111, 1220

Portegies Zwart, S.F. 2000, ApJ, 544, 437

Ransom, S.M. 2001, Ph.D. thesis, Harvard University

Sayer, R.W., Nice, D.J., \& Kaspi, V.M. 1996, ApJ, 461, 357

Voges, W. et al. 2000, IAU Circ., 7432, 1

White N.E., Giommi P., \& Angelini L. 2000, The WGACAT version of the ROSAT PSPC Catalogue, Rev. 1, (VizieR No. IX/31) 